\title{
Congestion control of mobile node in wireless mobile and communication using 802.15.4/ZigB ee
}

\author{
Dr.Aftab Ahmad Malik ${ }^{1}$ Aroosh Amjad ${ }^{2}$ Aleena Ayaz ${ }^{3}$ \\ ${ }^{1}$ Computer science, Lahore garrison university, University \\ Lahore, Punjab, Pakistan \\ ${ }^{2}$ Computer science, Lahore garrison university, University \\ Lahore, Punjab, Pakistan \\ ${ }^{3}$ Computer science, Lahore garrison university, University \\ Lahore, Punjab, Pakistan
}

\begin{abstract}
Clustering technique for load balancing tend to increase the stability of system and the communication between nodes in the system also improves. Many researchers have emphasized on the load in centroid as well as control the energy level of mobility in WSN. In case of consuming high load in cluster head that may on network and network gets down. Researchers have witnessed that load is an important factor in WSN 802.15.4 IEEE standard. This paper basically analyses load and its effectiveness in different scenarios (hexagonal shape of topology). increasing the number of end devices moreover by enabling and disabling the appropriate parameter on cluster tree it would be sorted out whether the load on cluster tree get high or not. The issue of load is being faced in the field of Wireless sensor network as well because the network has tiny sensor nodes and have limited battery life time, if the load increase the issue of battery consumption would be also increase. At last the result would be concluded without applying parameter and with parameter. Choosing the appropriate parameter, the great change is being witnessed in topologies
\end{abstract}

Keywords: Congestion, Coordinator, Clustering, ZigBee, IEEE 802.15.4.

\section{Introduction}

This paper is based on WSN (Wireless Sensor Network) that is famous on recent year researches. The used model is ZigBee 802.15.4 to build WSN. The architecture of WSN based on larger number of sensors nodes. The Wireless sensor act as RFD (Reduce Functional Devices) and FFD (Full Functional Devices). FFD is coordinator and routers and RFD are end-devices Topologies are the sequence or the pattern in which nodes and all connected lines are included. In this paper, the topology is based on analysis of

Congestion on Coordinators with ACK and Without ACK in increasing the number of end-devices and also Coordinator in mobility with $10 \mathrm{~km} / \mathrm{hrs}$ speed. The topology consists of end devices, routers and coordinators. The routers are placed in hexagonal shape and coordinator is in mobility. There are different number of end devices used in the topology. [1].

There are two topologies mesh and star used in 802.15.4/ZigBee, by using the two topologies and by deploying different number of end devices the load on the coordinator has been measured. In this topology, ACK parameter is used to identify the load/congestion on coordinator. The end-devices used in this topology does vary in two scenarios with ACK and without ACK. The reason of choosing [2].The parameter of ACKNOWLEDGEMET is that it is present in MAC layer. In physical layer, the transmission band used is 2.5 $\mathrm{GHz}$

Hexagonal shape of star and tree topologies are used to measure the congestion of devices by enabling and disabling of ACK. Two different topologies are used with four different scenarios to show the load of devices as well as the comparison between two topologies is shown in results to check which is the best among them and give efficient results on the basis of choosing right parameter. 
Congestion is an issue in a communication network, when there are an excessive amount of data packets are to be sent and received. In this case the performance of network gets down. In other words, congestion occur when there is a lot of weight on network. Let say. Amount of data packets are greater than the capacity of network etc. so congestion occur when too much traffic is on the route.

The capacity of output lines exceeds through the input traffic rate. If instantly, a block of data packets arrives on four or three input lines and all need the same output line, a queue will have built up in this case. The data packet will be missed if there is not enough record to grasp all the packets. But problem still exist if memory does increase. This why because, the packets have already timed out, by the time packets reach front of the queue. Source transmit duplicate data packets which are also added to queue when timer gets off. Thus, same packets increase the weight at all the way to its destination and are added to and forth. If the processors are slow, there would be great chance of congestion. Less speed of CPU in routers will perform the tasks very slowly such as updating table and queuing buffers. Witnessing this result, though there is excess line capacity queues are built up even then. This issue can be solved with high speed links, but this is not enough. Sometimes increment of bandwidth can deteriorate the problem of congestion as high bandwidth slow down the performance of network. Congestion can make itself worse.

If router does not have free buffers, it begins to ignore and discard the newly data packets which arrived. The sender may retransmit the data packet after the timer goes off when these packets are discarded. Packets are transmitted again and again by sender until the source gets the acknowledgment of these packets. Therefore, congestion takes place at the sending side due to multiple transmission of packets. Number of nodes which have different communication and computation capabilities exist in an Ad-hoc network. They have small batteries. This puts significant constraints on the power available for communication and computation.

Nodes in an ad-hoc network may have different capabilities of processing and battery levels within a decentralized and heterogeneous structure. in such cases, congestion problem can be take place, a node which is more powerful in form processing capacity can become idle, because it finishes its work quickly while others are occupies mostly and less powerful, consuming more energy. To increase the efficiency of applications and to solve the problem of congestion, Load balancing is one of the most important technique. The algorithms behind the load balancing are designed to distribute the load on the nodes equally, minimize the total task execution time and maximizing their utilization. There are many parameters if applied, congestion can be controlled and network can be more efficient. So, this paper concludes that by using the parameter such as ACKNOWLEDGEMT the congestion can be fully controlled.

\section{Acknowledgement}

ACKNOWLEDGMENT is name of signal in digital communication protocol, ACK declare that the packets has been received successfully at the destination end. After receiving the receipt of identifiable block of data with specific size, the signal of ACK is sent by receiving side back to the sending side. in order to be in identifiable form, the data block must conform to the protocol that is in use. When the client side receives the ACK packet from the server side, it transmits the data packet. Source either sends the packet or else ceases the transmission if the source fails to receive the Acknowledgement, it totally depends on the protocol. ACK is the form of signal embedded of an ASCII character, preserved for specific purpose. In some protocols, there are many ACK signals available that shows the data packet has been successfully received and recognition of specific commands, like power-down or standby. The acknowledgement imposed by the receiver may also affect congestion. Congestion may also have prevented NY slowing the sender side if the reciever does not acknowledge every packet it receives. Acknowledgment if added, it will increase the load on network. Thus, if there are less number of ACK are applied there would be less load on the network.For implementation there are several methods that can be used:

- If receiver has a data packet to be sent, only in that case a receiver may send an acknowledgement

- When a timer finished, then client side may send an ACK.

- A receiver may also decide to acknowledge only $N$ packets at a time.

Thus, ACKNOWLEDGEMNT as a parameter, is used in topology by enabling and disabling which shows the good and bad effect on network. All the simulation is done by using OPNET modeler, two topologies i.e. star and tree topology is used with end devices and coordinator having hexagonal shape and our chosen parameter is disabled in some scenarios to check the effect of disabling of ACK on the network. In some scenarios ACK is enabled to show how it badly effect the network and slow down the 
performance of network.For the maintenance and development of wireless and wired networks, IEEE supports several working groups. Let say, for cabled Ethernet 802.3 standard is used and 802.11 is developed for wireless Local area networks, also known as Wi-Fi. For instance, Bluetooth technology uses $802.15 .1,802.15 .3$ is a high-data-rate category for ultra-wideband (UWB) technologies, and 802.15.6 is for wireless body area networks (BAN). The 802.15 specifies a variety of wireless personal area networks for different applications. There are several others. The category 802.15.4 was developed to extend the life of low power consumption, to enhance the network as $802.15 .4 \mathrm{a} / \mathrm{b}$ uses. The category 802.15.4 was developed to extend the life of low power consumption, to enhance the network, for low data rate monitor. this was the basic standard with the recent updates are $802.15 .4 \mathrm{a} / \mathrm{b}$ with different countries like $802.15 .4 \mathrm{c}$ is developed for china, $802.15 .4 \mathrm{e}$ is developed for industry based applications, 802.15.4f for RFID and 802.15.4g is for smart utility networks [3] These all are special version that uses the base radio technology and protocol same as $802.15 .4 \mathrm{a} / \mathrm{b}$ uses the network, for low data rate monitor. this was the basic standard which shows recent updating are $802.15 .4 \mathrm{a} / \mathrm{b}$ with different countries like 802.15.4c for china, 802.15.4e for industrial application, 802.15.4f for RFID radio frequency identification and $802.15 .4 \mathrm{~g}$ is for smart utility networks (SUNs).[3] These all are special version that uses the base radio technology and protocol as same According to OSI layer of network operation, 802.15.4 standard is defined in physical and MAC layer. The PHY layer defines the wireless and wired connection, modulation techniques and power. The MAC layer has the complete format for the data handling purposes, the standard of 802.15.4 uses only the first two layers of OSI model with logical link control and service specific convergence sub layer, with the contact from all the upper layer of OSI which are defined by additional standards. There are three frequency ranges are provided, one is the $2.4 \mathrm{GHz}$ band and is the most widely used. And most known chips and modules us this band. The basic ambition of the standard is that, to provide the basic format through which other protocols and additional features can also be added to the upper most layers, the layer 3 to onward layer 7). [13]According to the ability of network, the much known standard 802.15.4 distributes two topologies. One is the star topology and other is mesh. Star topology is that in which there is one central device that can be hub, switch or router connected with all the other nodes in the topology. this standard also defines the peer to peer topology in which one node can connect to another node.. This basic topology may be divided into other topologies in the above network layers, like famous mesh topology.

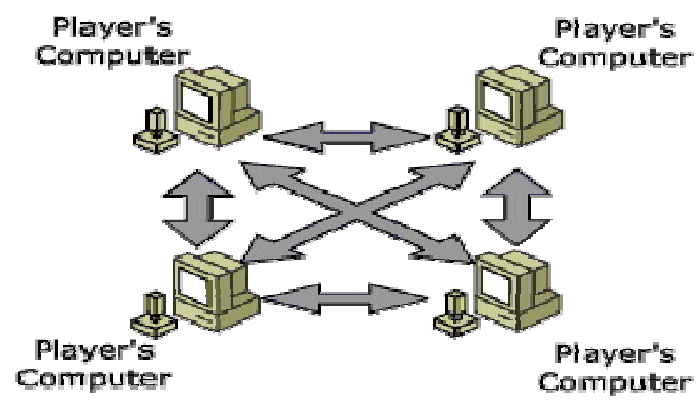

Fig-1

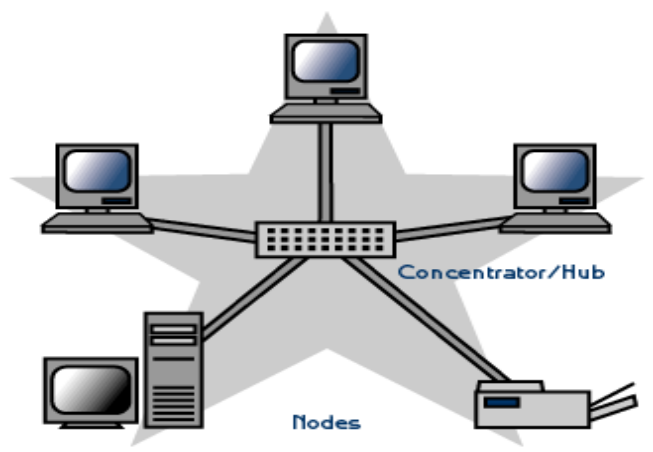

Fig-2

ZigBee uses the standard of IEEE 802.15.4 specification for ISO, physical and MAC layer. Standard of IEEE 802.15.4 offers four topologies like: star topology, tree topology, mesh topology and cluster tree topology. But ZigBee is the one which supports only star, tree and mesh topologies. As this paper involves ZigBee standard so the used topologies are discussed here:

Star topology Star topology: It consist of several nodes and one end device which can be either hub, switch or router. All the devices can communicate to the end devices. Main benefit of this topology is that its link never gets down but in the case of the failure or central device all the network gets down because all the data packets between devices must go through the center device so the central device may become bottlenecked. [3] Another benefit of star topology is that the data packets go through 
maximum two hops OR their communication occur between two hop counts.

Tree topology: in this topology, the network contains center device, many routers and end nodes. The main task of the router is to enhance the coverage of network. The end devices which communicated to the router or coordinator are said to be children nodes, routers and coordinator can be parent node. According to the fact. Only parent can have a child. An end device cannot have a child and cannot be a parent. [3] This is a specialty of tree topology. But it has drawbacks also. That if parent node gets down or disabled, the communication between them may get disturbed. One more thing is that if there are two nodes which are geographically connected and very close to each other, they cannot communicate directly.

Mesh Topology: in this topology, each infrastructure node and devices are connected with many network nodes. Each node is connected to all remaining nodes by using sperate cables. Mesh topology is very reliable and efficient but we cannot manage the nodes among the network. Mesh topology is specially used for home automation, smart HVAC control.

Cluster tree topology: This topology is an enhanced form of tree topology in which a cluster can be known as parent with its children, each cluster is identified by its cluster ID. IEEE 802.15.4 does not support this topology but ZigBee do so.

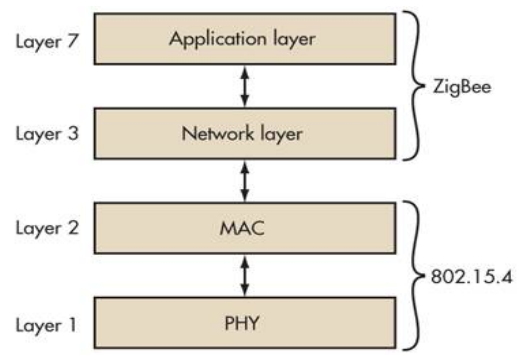

Fig-3 Zigbee and IEEE802.15.4 layer

The ZigBee is explained by layer 3 network layer and above the layer. It concatenate with the standard of 802.15.4 in layer 1 and 2

\section{ZigBee Technology}

Today there are a lot of high data rate communication standards that are used but none of these are able to meet the standards of sensors communication and control devices communication standard. Actually, the high data rate standards use low energy consumption and latency at lower bandwidths. [3]

ZigBee technology consumes low power and it costs low. ZigBee is best to make this kind of communication for home automation, industrial control and for several embedded applications and so on. [3]

ZigBee technology uses for control and sensor network on IEEE standard for WPANs (wireless personal area network). This is one of the product of ZigBee alliance. ZigBee standard defines the MAC and physical layer to handle devices at lower data rates. It operates at 868 $\mathrm{MHz}$, and $2.4 \mathrm{GHz}$ frequencies. ZigBee communication is cheaper than other short- range wireless senor networks. It allows lots of nodes to connect each other for built WAN (Wide area network).

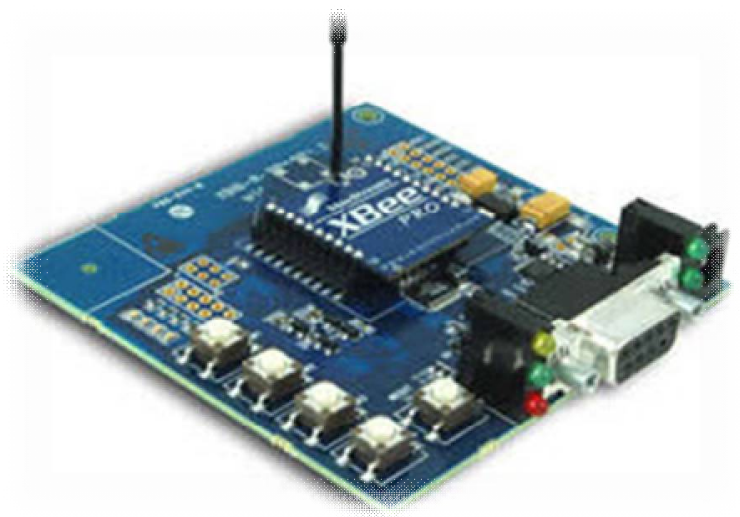

Fig-4

There are three different type of devices that are used to structure the zig ZigBee bee system.

ZigBee Coordinator: ZigBee coordinator have a responsibility to attach with network and choosing the key network parameter. It acts like a root of the network. ZigBee coordinator takes the responsibility for storing and handling the information while performing the operations for example: receiving and storing the information.

ZigBee Router: ZigBee routers are devices capable of routing data. It acts like the intermediary devices that allows the data to forward to and from through them to other nodes.

End Devices: These devices in ZigBee structure have minimum functionality to connect with the parent nodes, 
because these devices have no routing capability. In wireless sensor network, the function of end devices is not directly suited for either type of nodes -the sink or sensor. [3]

In ZigBee structure the different number of devices depends on the type of network like

$>$ Star topology

$>$ Tree topology

$>$ Mesh topology networks.

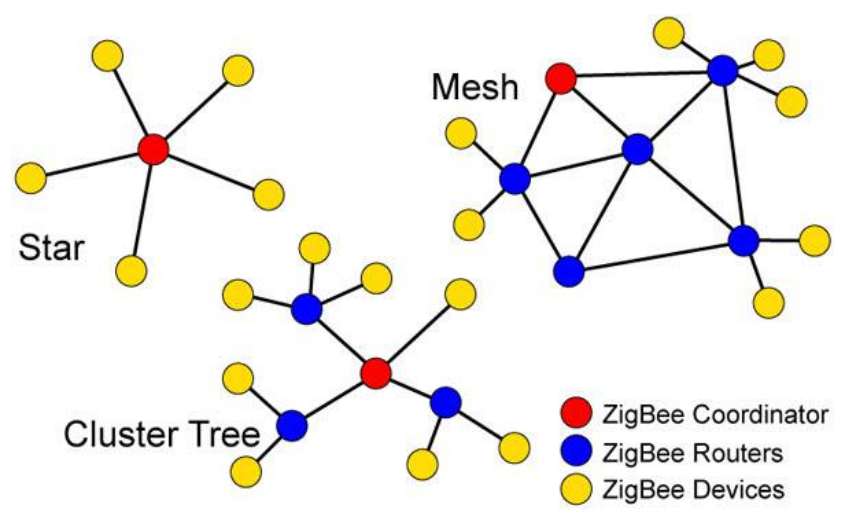

3.1 ZigBee Protocol Architecture

There are various types of layers which are define in the ZigBee architecture.

- Physical Layer: physical layer is responsible for modulation and demodulation technique. Modulation for the going signal and demodulation for the incoming signals. Basically, it transmits and receives information from a source.

- MAC layer: It access the network with collision avoidance by using carrier-sense.

- Network layer: This layer in ZigBee protocol architecture supports sublayer. This is situated between Mac layer and application layer.

- Application Support Sublayer (APS): This Layer provides the best services to connect with the network layer for data and management services. APs define the unified communication structure.

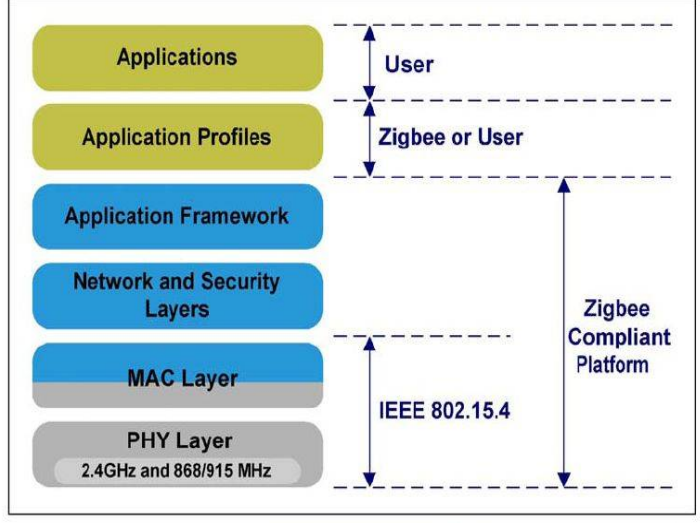

Silicon

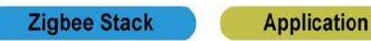

Fig-3.2

\section{ZigBee Technology Application \\ * Home automation system \\ * Industrial Automation system \\ * Smart Monitoring system \\ 4 Smart Grid M onitoring}

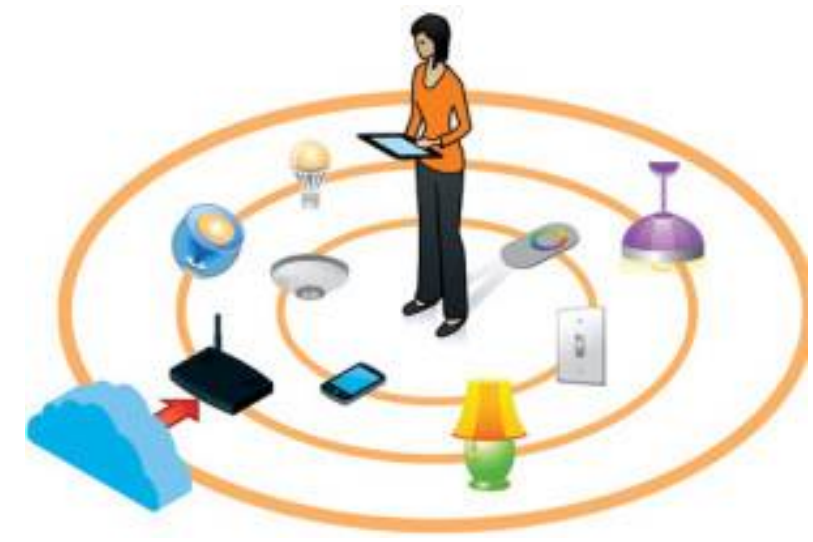

Fig-3.3

\section{Literature View}

In cluster, independent nodes are combined in a uniform system through networking and software. Typically, as compare to the single computer clustering mechanism provide reliability to provide better computational power. Clusters linked together by dedicated network that is of a high-speed.

Cluster is categorized in four categories. 
- High-availability Cluster

- Load balancing Cluster

- High performance Cluster

- Grid Cluster

In our research, we will use the load balancing cluster to control the congestion of mobile nodes in wireless sensor networks. This type of cluster distributes the information among multiple nodes running the same program or having the same information. Load-balancing cluster used to increase the reliability and reduces the congestion between sensor nodes in wireless network.

The mechanism of clustering provides a very comfortable way for managing resources. It may having many important qualities like separation of code, accessing to channel, techniques of routing and control power. There are centroids in clustering that are responsible for routing node messages within each cluster during the distribution of services. For efficient communication between nodes that are supported by cluster heads. If there is a lot of load on the cluster heads, the performance of other protocols will be decrease which is based on clustering.

Many publishers discussed the clustering selection's base of cluster head factors like ID, connectivity degree and randomized. Mostly the selection of cluster-head is preferred, if the changing of topology or the load shared between different nodes, clustering like low / high connectivity are mostly applied. Centroid will be picked as from the nodes present between a lots of nodes. But in the result its energy drains very fast. Moreover, many researchers researched that protocols for clustering load balancing is not considered within clusters to variable density of nodes in system. If load is not equal or balance in system then there must be cluster with very high or very low density.

Main purpose of this paper [13] research is to Cluster the wireless sensor network load balance efficiency among high energy gateway nodes. As node clustering is a very well-known research area. Clustering is an efficient technique which increase the stability of network in large number of nodes by allowing the node to save energy and thus extend the life time of network through the operation of clustering and with the help of balancing the weight between the gateway nodes. The author actually wanted to approach the load balance among clusters and also tries to cluster the sensor node as close to high energy cluster heads. To define the load-balancing 'effects in clusters, author has integrated clustering technique with 6 different routing approaches.
Many simulations are performed by using hundred sensor nodes. Author used different metrics to evaluate the performance of routing algorithms. Recent research work in the field of load balancing in different areas, the basic purpose of all the researches is to increase the life of cluster head and improves the network efficiency. Clustering is a mechanism in which two or more computer works as a single computer and it is specially used in load balancing, fault tolerance and parallel Processing. [12] Every cluster in a network has a centroid that is used to divide the data packets in its cluster. Each cluster have a cluster size that is actually the nodes in that cluster. Cluster size is not fixed because it depends on the active nodes in a cluster as nodes move from one cluster to another cluster, in this case if a cluster Have too many active nodes then it became overloaded. Due to this the cluster head are under loaded. They lose their battery powers faster. Overloaded cluster heads actually cause the low performance of the network.

On the Behalf of the research studies a new implemented algorithm is proposed to increase the network life time through equally distributing the load among cluster heads. Algorithm uses parameter as energy threshold. [13] From which one uses to balance the load by distributing it equally among the cluster-heads, although second one is used to trigger local re-clustering in network.

One more algorithm from another research is cross-layer based load balance, which main purpose is to divide and balance the network load between the whole network nodes in order to reduce the delay and low efficiency of network by merging and resizing the cluster or forming new cluster in network. In 2011 people do lot of research on load balancing in different areas like Load blanc in ad hoc network, load balance in wireless sensor network, Avoiding the collision among the nodes by sharing the equally load balance. Actually, the main purpose of different research is to

- Equally distribute the load on the Number of nodes among clusters.

- To balance the load in the network traffic.

- To balance the cluster-head's load between its members.

In [3], the purpose is to increase the network performance by purposing the cluster head load balance technique (CLBT), because fast connectivity among nodes is supported by cluster-heads. This CBLT technique is used dynamic transmission rate to redistribute the load among cluster-heads in MANET. High load in cluster-heads 
share the load between nodes equally. High loaded cluster-heads decreases the transmission range and low loaded or unloaded cluster-heads increases the transmission range. [11] The CLBT technique uses two levels.

Local Level between cluster-heads and general level between cluster-heads and its near cluster-heads.

In general level every cluster-head includes a parameter and its current and predefined load. Every cluster-head compares its parameter load with other cluster-head parameter load, if the load is exceeding then cluster-heads uses the CLBT algorithm. And then same loaded clusterheads like high or low loaded again distribute the load among them.

In local level, every node has a parameter, its load and its position. A node sends message to its cluster head containing its load and position. All existing clustering algorithm does not consider the nodes which are active after a cluster created under some traffic load's circumstances of traffic load and underload cluster head may occur as a result. To ignore this issue, this author introduced CLBT that that uses transmissions range dynamically to divide the load equally between centroids head. CLBT works when all the cluster heads are presents either it is loaded or not, [10] CLBT initiate the loadbalance among cluster-heads using the transmission range to equally redistribute their nodes. Cluster heads which are loaded reduces its range of transmitting data till it dismiss the node which are active in its cluster. Same as, unloaded cluster-head increases its transmission range to include the nodes which are active in the cluster

\section{Methodology}

Congestion control of mobile node in wireless mobile and communication through IEEE 802.15.4/ZigBee

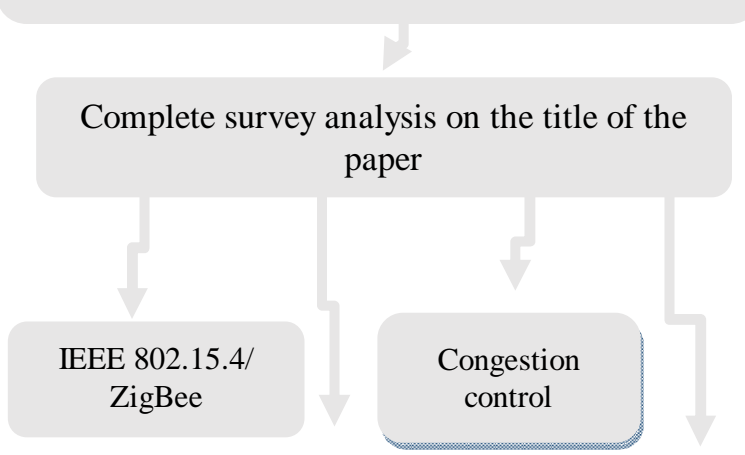

Mobile-nodes and coordinator

Verification Procedure

\section{Load-Balancing Clustering}

The main goal of this approach is to cluster network efficiently around the gateway node and with different topologies. Cluster increase the network scalability and also increase life of network by allowing communication with close nodes and by balancing the load among gateway. Clustering made with cost of communication and depend upon the load on cluster. [5]

Ad-hoc network is combination of wireless nodes without any centralized structure. To distribute service and route packets in its cluster the network is divided into clusters, each Cluster has a cluster head. Due to difference between the levels of nodes the network may highly load or lightly loaded. The service distribution and routing mechanism are both based on the cluster's architecture and this may debase the performance. To distribute the load between the cluster heads, a new technique is proposed which is called Cluster Head Load-Balancing Technique (CLBT). CLBT will be invoked to initiate load-balancing between cluster heads by adapting the cluster heads transmission range to fairly redistribute their respective nodes when loaded and unloaded cluster heads are present. , unloaded cluster head gradually raises its transmission range to include active nodes simultaneously loaded cluster head reduces its transmission range until it dismisses the active nodes from its cluster head [9].

Clustering is the most important mechanism for ad-hoc network especially wireless sensor network. it help to lessen the complexity with the management of data which is related to the mobile nodes any with the Lessing of load in network the network. After the development of clusters, every cluster have a specific size according to its energy level and the transmission range of centroid. The size of cluster head OR centroid is not fixed it may vary. Because every node is on mobility and can move from on cluster to another cluster. If there are too much active nodes present in between the one cluster then cluster might be overloaded. the battery level of the nodes is very less so cluster head may lose its energy level very soon, which may cause a reflection of centroid and many data sets exchange within the nodes may results in high cost and computation overhead. If the distribution among the nodes and routing mechanism is based on cluster's architecture then the distribution and routing performance 
will be also affected by the clusters which are overloaded and overloaded centroids cause the problem and decrease the lifetime of network and network goes down. [5]

\section{Experimental Work}

\section{Scenario 1:}

This table shows the parameter that are used in the topology.

\section{Table.1}

In this scenario, there is one coordinator and 6 routers used in hexagonal shape and 12 end-devices with ACK parameter and also add without ACK topology as it is used in $2^{\text {nd }}$ scenario.

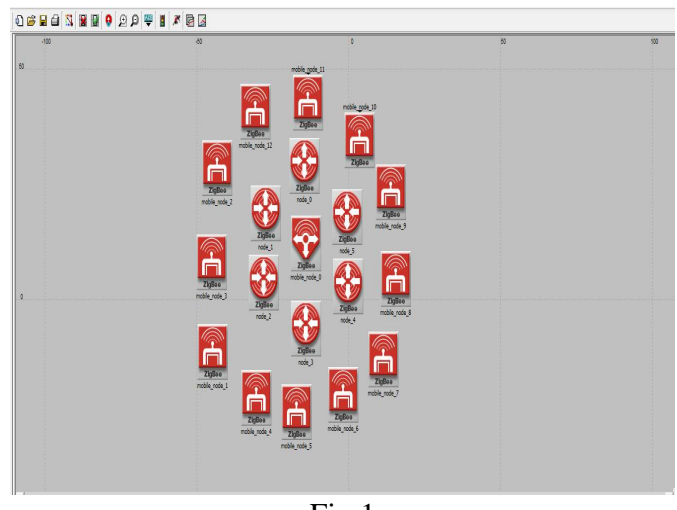

Fig. 1

Hexagonal shape 12 nodes topology

\section{Scenario 2:}

\begin{tabular}{|c|c|}
\hline Parameters & Values \\
\hline ACK mechanism & OFF \\
\hline Status & Enable \\
\hline
\end{tabular}

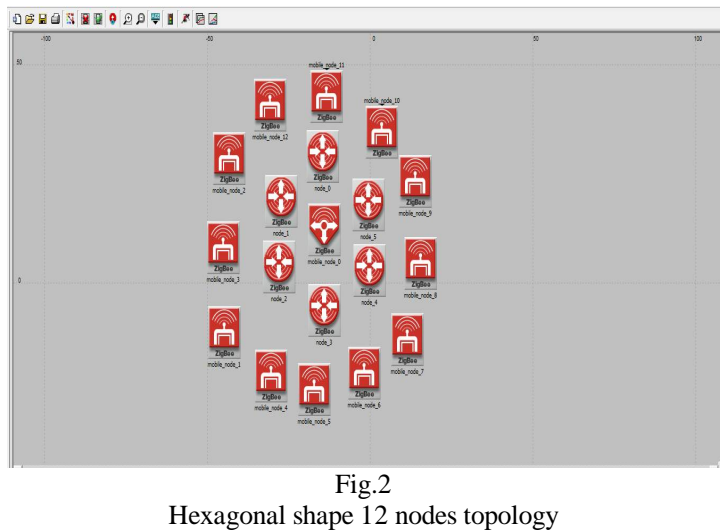

\begin{tabular}{|c|c|c|}
\hline \multirow{3}{*}{$\begin{array}{l}\text { In this } \\
\text { we used }\end{array}$} & Parameters & Values \\
\hline & ACK mechanism & $\mathrm{ON}$ \\
\hline & Status & Enable \\
\hline
\end{tabular}

and 6 routers

used in hexagonal shape and 12 end-devices but without ACK parameter mean ACK parameter is off.

\section{Scenario 3:}

\begin{tabular}{|c|c|}
\hline Parameters & Values \\
\hline ACK mechanism & ON \\
\hline Status & Enable \\
\hline
\end{tabular}

Table.3

In $3^{\text {rd }}$ scenario, there is one coordinator and 6 routers in hexagonal shape and 22 end-devices with ACK parameter. We analysis on load parameter of both topologies that which topology is have high load on with $\mathrm{ACK}$ or without ACK.

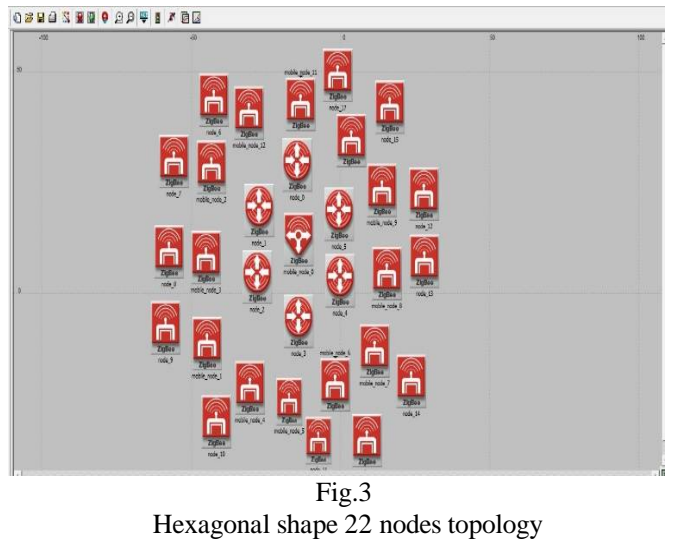




\section{Scenario 4:}

\begin{tabular}{|c|c|}
\hline Parameters & Values \\
\hline ACK mechanism & OFF \\
\hline Status & Enable \\
\hline
\end{tabular}

Table.4

In $\quad 4^{\text {th }}$

there is one coordinator and 6 routers in hexagonal shape and 22 end-devices without ACK parameter. We analysis on load parameter of both topologies that which topology is have high load on with ACK or without ACK.

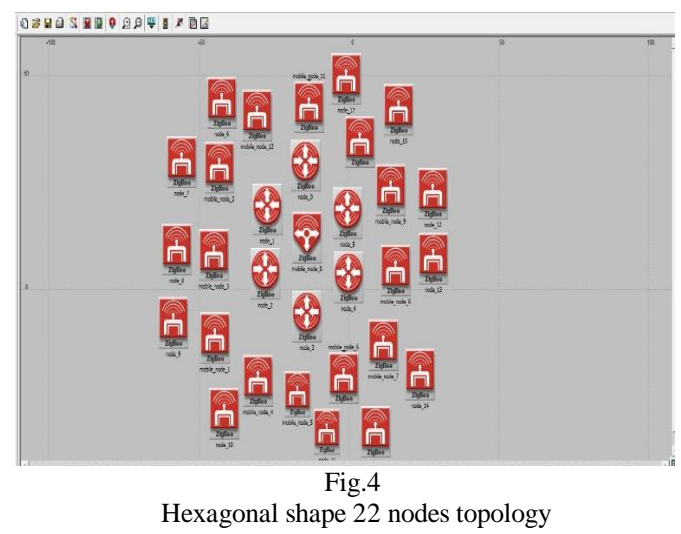

Results:

In result section, we will show you the results of topology using ZigBee 802.15.4 OP-Net simulator that which topology has maximum load with ACK or without ACK.

\section{Scenario}

The load on coordinator is high in with ACK because the end-devices is 12 and he send data to router and router further send to coordinator. After data receive at destination the coordinator also sends ACK to sender side during these processes the other packet wait minor time instead of without ACK topology.

But in ACK the load is high because when sender side send data to other side it will take time because of ACK, the ACK send receiver side to sender that he receives the data so the other packet or data wait until the first data is being properly process or done his job.

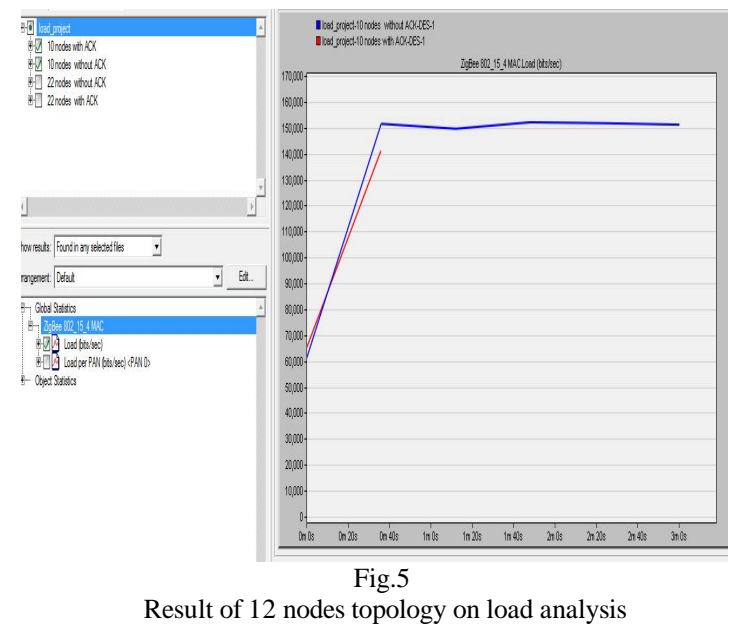

You can see the result in this figure that blue line shows the result of without ACK and red line show the load with ACK parameter. This figure include the two toplogy result.

\section{Scenario 2:}

The load on coordinator is high in with ACK because the end-devices is 12 and he send data to router and router further send to coordinator. After data receive at destination the coordinator also sends ACK to sender side during these processes the other packet wait minor time instead of without ACK topology.

But in ACK the load is high because when sender side send data to other side it will take time because of ACK, the ACK send receiver side to sender that he receives the data so the other packet or data wait until the first data is being properly process or done his job.

You can see the result in this figure that blue line shows the result of without ACK and red line show the load with ACK parameter. This figure include the two toplogy result.

\section{Scenario 3:}

The final output of 3 scenario on load is given below. In this result, the load on coordinator is show on both topologies that we used in this paper (12 node and 22 nodes). The final output will show in fig.7. 


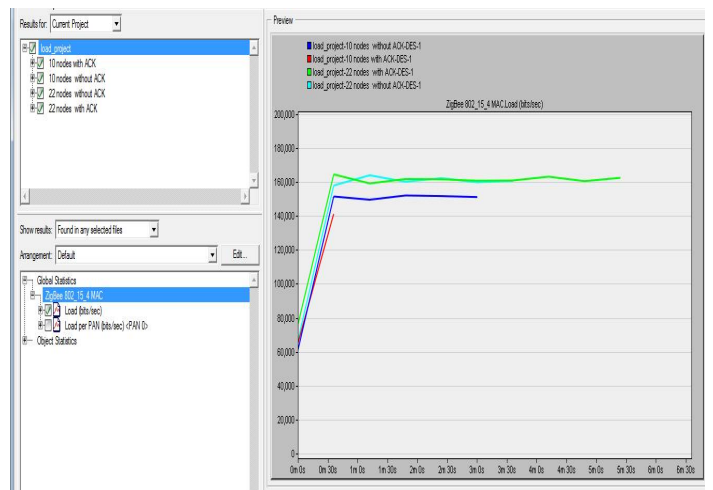

Fig.7

Result of 12 and 22 nodes topology on load analysis

\section{Conclusions}

In this paper we concluded the performance of choose parameter which is ACKNOWLEDGEMENT, on the behalf of this appropriate parameter we came to know that congestion can be controlled. We have deployed end devices and coordinator on OPNET by using different topologies, thus by enabling the chosen parameter we came to know that the congestion on the network is excessive, but without Acknowledgement it has been proved that congestion OR load is decreased within the network. The above scenarios have shown excellent result on the basis of congestion

\section{Acknowledgments}

The authors are great full to the vice chancellor of Lahore Garrison University for encouragement

\section{References}

[1] Anju, S. T. (2013). Energy Efficient Clustering In Wireless Sensor Network: A Review. 3.

[2] Ashraf A. M. Khalaf, M. S. (2016). Effects of ZigBee Component Failure on the WSN. ICM, 4.

[3] InformIT. (2009, december 2). Retrieved from www.informIT.com:http://www.informit.com/articles/article. aspx?p=1409785\&seqNum $=4$

[4] Jun Luo, L. J. (2007). Performance Analysis of Synchronous Wakeup Patterns in Contention. IEEE, 5.

[5] Labdah ALGhafran, Z. B. (2013). Load-Balancing Technique in Clustered Mobile Ad-Hoc Networks. International Conference on Advanced Computer Science Applications and Technologies, 4.
[6] Marina Petrova, J. R. (n.d.). Performance Study of IEEE 802.15.4 Using measurements and simulations. 6 .

[7] Mikko Kohvakka, M. K. (2006). Performance Analysis of IEEE 802.15.4 and ZigBee. ACM, 10.

[8] N. Vlajic, D. S. (2009). Performance Analysis of ZigBeebased Wireless Sensor Networks. Third International Conference on Sensor Technologies and Applications, 8.

[9] Poole, I. (2011, march 12). wireless connectivity. Retrieved from radio_electronics.com: http://www.radioelectronics.com/info/wireless/zigbee/zigbee.php

[10] Rachida Aoudjit, M. L. (2009). Load Balancing: An Approach Based on clustering on Ad-hoc network. Journal of Computing and Information Technology - CIT 17, 2009, $2,177-184,8$.

[11] THAKUR, D. (2009, january 5). Computer notes. Retrieved fromecomputernotes:http://ecomputernotes.com/computernet wor kingnotes/communication-networks/what-is-congestioncontrol- describe-the-congestion-control-algorithmcommonly-used

[12] Younis, G. G. (2013). Performance Evaluation of LoadBalanced Clustering of Wireless. IEEE, 7. 